79 IS PRIMARY CHEMORADIATION A BETTER TREATMENT? A RETROSPECTIVE STUDY OF EARLY STAGE NODEPOSITIVE CERVICAL CANCER

Nan Zhang, Hong Zheng. Gynecology Department, Peking University Cancer Hospital and Institute, Key laboratory of Carcinogenesis and Translational Research, Ministry of Education of People's Republic of China, Beijing 100142, China

\subsection{6/ijgc-2020-ESGO.8}

Introduction/Background Cervical cancer is the second most frequently diagnosed cancer and the third leading cause of cancer death for women in developing countries. Radical hysterectomy with bilateral pelvic lymph node dissection is usually preferred for patients of stage IB1-IIA2. Currently, image examinations have certain limitations in diagnose of lymph node metastasis and their detection accuracies are not satisfactory. Only the pathological examination after removal of the suspected metastatic lymph nodes during surgery can conclusively identify the presence of metastasis. If there is a positive result of lymphatic metastasis, there is no clear guideline whether to complete a radical surgery, or to only conduct a systematic lymphadenectomy, followed with adjuvant Concurrent Chemoradiotherapy (CCRT). This retrospective study aimed to compare the efficacy and safety of the two treatment modalities.

Methodology 49 stage IB1-IIA2 cervical cancer patients with lymphatic metastasis confirmed by systemic pelvic and paraaortic lymph node dissection from 2007 to 2018 were reviewed. The patients were treated with either primary chemoradiation or radical hysterectomy followed by adjuvant chemoradiation after lymphadenectomy. Survival states and adverse events of the two treatments were compared.

Results Median follow-up time was 45 (range 11-119 months) months. In non-radical surgery group, 1 patient $(1 / 15,6.7 \%)$ relapsed and died, while in radical surgery group, 7 patients $(7 / 27,25.9 \%)$ relapsed and $5(5 / 27,18.5 \%)$ died. Significant difference was found in the mean progression-free survival between the two groups, which was 69(95\%CI 49.11888.882 ) months in non-radical surgery group and $44(95 \% \mathrm{CI}$ $35.857-52.143)$ months in radical surgery group $(p<0.01)$. There was significant difference in three-year progression-free survival $(86 \%$ vs. $71 \%, \mathrm{p}<0.01)$. Grade $3-4$ toxicity was comparable between the two groups $(26.7 \%$ vs. $25.9 \%, p=0.958)$.

Conclusion For stage IB1-IIA2 cervical cancer patients with positive lymph node, primary chemoradiation after pelvic and para-aortic lymphadenectomy seems to have better survival outcomes compared with radical hysterectomy by laparoscopy plus chemoradiation in the retrospective study with limited cases. Evidence from a randomized controlled study is in need to confirm the optimal treatment for early stage node-positive cervical cancer.

Disclosures The authors declare that they have no conflicts of interest.

\section{USP18 PROMOTES CELL PROLIFERATION AND SUPPRESSED APOPTOSIS IN CERVICAL CANCER CELLS VIA AKT SIGNALING PATHWAY}

${ }^{1}$ Wenjing Tellydiao, ${ }^{1}$ Qisang Guo, ${ }^{1}$ Caiying Zhu, ${ }^{1} Y u$ Song, ${ }^{1}$ Hua Feng, ${ }^{1}$ Yuankui Cao, ${ }^{1}$ Ming Du, ${ }^{2}$ Huifen Chen. 'Obstetrics and Gynecology Hospital of Fudan University; ${ }^{2}$ Shanghai First Maternity and Infant Hospital, Tongji University School of Medicine

10.1136/ijgc-2020-ESGO.9
Introduction/Background Cervical cancer is one of the most common malignancies in women worldwide. USP18 (USP43), a member of Ubiquitin-specific protease family, has been linked to several human malignancies except cervical cancer. The current study aimed to explore the expression and possible role in cervical cancer.

Methodology Real-time PCR and immunohistochemical staining was performed to analyze USP18 expression in cervical cancer tissues and normal tissues. USP18 expression was manipulated in cervical cancer cell lines, and its biological function in cell proliferation and apoptosis was assessed by Cell Counting Kit-8 assay and Annexin V/PI staining, respectively.

Results We demonstrated that USP18 expression was increased in cervical cancer specimens and cell lines. Knocking down of USP18 in cervical cancer cell lines, SiHa and Caski, inhibited cell proliferation, while induced apoptosis and the expression of cleaved caspase-3. On the contrary, USP18 overexpression showed reversed effects in Hela cells. Moreover, Gene Set Enrichment Analysis showed that USP18 expression level was correlated with PI3K/AKT signaling pathway in cervical cancer. Further, the PI3K/Akt inhibitor LY294002 blocked the effects of USP18 overexpression on cervical cancer cells.

Conclusion The current study indicates the oncogenic role of USP18 in cervical cancer, which will deepen the understanding in the pathogenesis of cervical cancer and may provide a novel target for cancer therapy.

\section{INFLUENCE OF INITIAL VOLUME OF CERVICAL CANCER ON ACHIEVING THE RECOMMENDED BRACHYTHERAPY DOSE AT TARGET VOLUMES}

${ }^{1}$ Aleksandar Tomasevic ${ }^{1}$ Vesna Plešinac Karapandžić ${ }^{2}$ Sandra Vučković, ${ }^{2}$ Dragoslava Marjanović, ${ }^{2}$ Mirjana Miković, ${ }^{2}$ Marko Radović, ${ }^{3}$ Milena Tomašević. 'Institute of Oncology and Radiology of Serbia; University School of Medicine; ${ }^{2}$ Institute of Oncology and Radiology of Serbia; ${ }^{3}$ Institute for Student's Health of Belgrade University

\subsection{6/ijgc-2020-ESG0.10}

Introduction/Background 3D brachytherapy (BRT) planning, based on magnetic resonance (MR) imaging, has become a standard approach in cervical cancer radiotherapy treatment in many radiotherapy centres. T2W MR images give us precise visualization of the tumor tissue volume and tumor changes during the treatment. Adequate BRT dose coverage of the target volumes, presented through D90 and D100 dose parameters, has a primary role in achieving local control of the disease.

The goal is to estimate the initial tumor volume impact on the registered target volume dose parameters.

Methodology The curative chemoradiation was applied to 30 patients with advanced cervical cancer. Brachytherapy was performed in a high-dose rate regimen, in 4-5 weekly applications, with a dose of 7Gy per application, starting after 15 external beam fractions, endocavitary without interstitial catheters. For each patient a 3D T2W MR imaging was performed, initially prior treatment and at the time of the first and the fourth BRT application. MR images were used for tumor volume assessment and for 3D BRT planning, obtaining that way the target dose-volume parameters. Initial tumor volume influence on the registered HR-CTV dose parameters (clinically the 
most important target volume dose parameter) was then investigated.

Results MR based assessment of tumor volumes initially, at the time of the first and the fourth BRT application, were as follows: $(49.9 \pm 33.3 \mathrm{ccm}, 11.3-124.2 \mathrm{ccm}),(17.3 \pm 19.2 \mathrm{ccm}$, $1.7-78.4 \mathrm{ccm})$ and $(7.0 \pm 10.9 \mathrm{ccm}, 0.8-58.5 \mathrm{ccm})$ respectively. Registered EQD2 HR-CTV doses for the whole group of patients were D90 (107.15 $\pm 22.06 \mathrm{~Gy})$, and D100 (80.66 $\pm 14.58 \mathrm{~Gy})$. Initial tumor volume showed a strong negative, statistically significant correlation with registered HR-CTV D90 dose at the time of the first BRT application (rho= 0.8 ). ROC analysis confirmed the discriminative influence of initial tumor volume on achieving recommended HR-CTV dose, with the best initial tumor volume cut-off value of $47.87 \mathrm{ccm}$, with high sensitivity $89.4 \%$, and specificity $90.9 \%$. A statistically significant difference between two groups of patients (regarding the identified tumor volume cut-off value of $47.87 \mathrm{ccm}$ ) was found for the EQD2 HR-CTV doses: D90 (120.4 $\pm 14.6 \mathrm{~Gy}$ vs $87.33 \pm 15.48 \mathrm{~Gy}, \mathrm{p}=6.9 * 10-7)$ and D100 $(101.8 \pm 13.39$ vs $75.85 \pm 11.93 \mathrm{~Gy}, \mathrm{p}=9 * 10-5)$.

Conclusion The initial volume of the cervical tumor has a great influence on achieving the recommended values of HRCTV D90 and D100 doses. Tumors with initial volume greater than $47.87 \mathrm{ccm}$ cause a statistically significant lower total EQD2 dose at the HR-CTV volume, leading to poor local disease control.

Disclosures The results are part of my paper published recently in $\mathrm{J}$ BUON.

https://jbuon.com/archive/25-1-108.pdf

\section{DISEASE-FREE SURVIVAL IN EARLY-STAGE CERVICAL CANCER: COMPARISON OF LAPAROSCOPIC VERSUS OPEN SURGERY IN A TERTIARY CENTRE}

Amanda Veiga-Fernández, María López-Altuna, Ignacio Romero-Martínez, Elsa Mendizábal Vicente, Patricia Rincon Olbes, Santiago Lizarraga Bonelli.

\subsection{6/ijgc-2020-ESG0.11}

Introduction/Background Over the last years, minimally invasive surgery was advancing as the preferred approach in many cancer centres for the treatment of early-stage cervical cancer, as it offers advantages against open abdominal surgery in inhospital and short-term outcomes. However, results from a recent randomized trial suggested lower rates of disease-free survival in the follow-up of patients operated with the minimally invasive approach.

The aim of our study was to compare, in our tertiary centre, the rate of disease-free survival at 3.5 years between both surgical techniques.

Methodology Retrospective single-centre study including patients who underwent total laparoscopic radical hysterectomy (LRH) or open radical hysterectomy (ORH) due to early-stage cervical cancer (IA1-IIA1) between 2005-2017.

Results A total of 63 patients were included (39 LRH and $24 \mathrm{ORH})$. Baseline characteristics are described in table 1. Mean age was similar in both groups $47.5 \pm 13.3$ vs. 48.3 $\pm 12.6(\mathrm{p}=0.8)$. The prevalence of high-risk HPV was similar in both groups with a higher rate of prior conization in the LRH group $22(56.4 \%)$ vs. $5 \quad(20.8 \%) \quad(p=0.006)$. There were no significant differences between both groups in terms of parametrial involvement, histologic subtypes and stage of disease. In almost half of the patients in the LRH group the uterine mobilizer was used during surgery, with 1 case of uterine perforation. There were no significant differences at 3.5 years follow-up in terms of recurrences [LRH 4 (10.3\%) vs. ORH $2(8.3 \%)(p=0.8)]$ and overall death (being secondary to their oncological process in all cases), $2(5.1 \%)$ vs. 1 $(4.2 \%) \quad(p=0.87)$. Kaplan-Meier analysis revealed a similar rate of disease-free survival at 3.5 years in both groups: LRH $87.2 \%$ vs. $87.5 \%(p=0.95)$ (figure 1$)$. The rate of disease-free survival in patients in which the uterine mobilizer was used was $100 \%$.

We hypothesized that, in experienced hands and with appropriate patient selection, a minimally invasive approach via laparoscopic surgery can be as effective as conventional

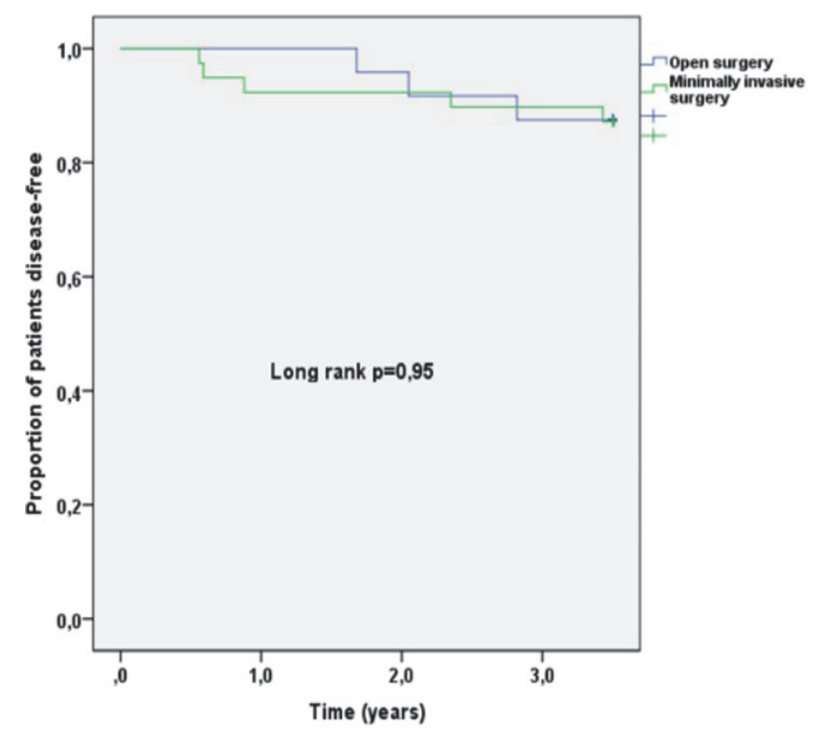

Abstract 154 Figure 1

\section{Abstract 154 Table 1}

\begin{tabular}{|l|l|l|l|}
\hline & $\begin{array}{l}\text { Laparoscopic radical } \\
\text { hysterectomy } \\
\mathrm{N}=39\end{array}$ & $\begin{array}{l}\text { Open radical } \\
\text { hysterectomy } \\
\mathrm{N}: 24\end{array}$ & \\
\hline Age (years) & $47,5 \pm 13,3$ & $48,3 \pm 12,6$ & $\mathrm{P}=0,8$ \\
\hline Prior conization & $22(56,4 \%)$ & $5(20,8 \%)$ & $\mathrm{P}=0,006$ \\
\hline High-risk HPV & $7(17,9 \%)$ & $1(4,2 \%)$ & $\mathrm{P}=0,2$ \\
\hline Parametrial involvement & $1(2,6 \%)$ & $2(8,3 \%)$ & $\mathrm{P}=0,3$ \\
\hline Histologic subtype & & $13(54,2 \%)$ & $\mathrm{P}=0,1$ \\
Squamous-cell carcinoma & $13(33,3 \%)$ & $6(25 \%)$ & \\
Adenocarcinoma & $13(33,3 \%)$ & $1(4,2 \%)$ & \\
Adenosquamous carcinoma & 0 & $3(12,4 \%)$ & \\
Other & $12(30,8 \%)$ & $1(4,2 \%)$ & \\
Not reported & $1(2,6 \%)$ & $7(29,2 \%)$ & \\
\hline Stage of disease & & $17(70,8 \%)$ & \\
IA1,IA2 & $14(35,9 \%)$ & 0 & \\
IB1,IB2 & $24(61,5 \%)$ & & \\
IIA1 & $1(2,6 \%)$ & $\mathrm{P}=0,6$ \\
\hline Use of uterine mobilizer & & 0 & \\
during surgery & & $24(100 \%)$ & \\
Yes & $18(47,4 \%)$ & 0 & \\
No & $9(23,7 \%)$ & $\mathrm{P}=1$ \\
Unknown & $11(28,9 \%)$ & $16(66,7 \%)$ & \\
\hline Uterine perforation during & $1(2,6 \%)$ & $6(25 \%)$ & \\
surgery & & $2(8,3 \%)$ & \\
\hline Adjuvant therapy & & & \\
No & $30(76,9 \%)$ & $7(17,9 \%)$ & \\
Radiotherapy & $2(8,3 \%)$ & & \\
Radiotherapy and & & & \\
Chemotherapy & & & \\
\hline
\end{tabular}

